\title{
An approach to the patient with a suspected tachycardia in the emergency department
}

\author{
A Chin, ${ }^{1}$ MB ChB, MPhil, FHRS; B Vezi, ${ }^{2}$ MB ChB, FCP (SA), Cert Cardiology (SA); M Namane, ${ }^{3}$ MB ChB, MPhil; \\ H Weich, ${ }^{4}$ MB ChB, MRCP (UK), MMed (Int), Cert Cardiology (SA); R Scott-Millar, ${ }^{1}$ MB BCh, FCP (SA) \\ ${ }^{1}$ Division of Cardiology, Department of Medicine, Faculty of Health Sciences, Groote Schuur Hospital, University of Cape Town, South Africa \\ ${ }^{2}$ Ethekwini Hospital, Durban, South Africa \\ ${ }^{3}$ Vanguard Community Health Centre, Cape Town, South Africa \\ ${ }^{4}$ Division of Cardiology, Department of Medicine, Faculty of Medicine and Health Sciences, Tygerberg Hospital, Stellenbosch University, Cape Town, \\ South Africa
}

Corresponding author: A Chin (ashley.chin@uct.ac.za)

Patients present to the emergency department with either an ongoing tachycardia or a history suspicious of a tachycardia. Either way, the tachycardia needs to be documented, preferably on a 12-lead electrocardiogram (ECG) for diagnosis and management. If a tachycardia is not documented, a careful history of the palpitations should be taken to see if further monitoring and investigations are required. If a tachycardia is confirmed on an ECG, the clinician needs to classify it according to two variables: (i) regularity of the rhythm; and (ii) QRS width. This will allow a differential diagnosis to be made.

S Afr Med J 2016;106(3):246-250. DOI:10.7196/SAMJ.2016.v106i3.10322

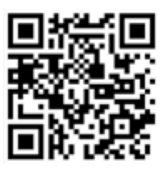

Patients may present to the emergency department with either an ongoing tachycardia or a history suspicious of a tachycardia. In either case, the tachycardia needs to be documented, preferably on a 12-lead electrocardiogram (ECG) for diagnosis and management. If a tachycardia is not documented, a careful history of the palpitations should be taken to establish if further monitoring and investigations are required. If a tachycardia is confirmed on an ECG, the clinician needs to classify it according to two variables: (i) regularity of the rhythm; and (ii) QRS width. This will enable a differential diagnosis to be made (Fig. 1). A narrow QRS or supraventricular tachycardia (SVT) can be caused by any arrhythmia that arises above the level of the bifurcation of the bundle of His. A wide QRS tachycardia has a differential diagnosis, but by far the most common cause of a wide complex tachycardia (WCT) is ventricular tachycardia (VT). The ability of the clinician to distinguish between an SVT and a VT is crucial, as the management of these conditions differ and inappropriate management of the tachycardia may have lethal consequences. In a busy emergency department, such patients need to be appropriately triaged. In this review we focus on an approach to a patient with a tachycardia, who presents to the emergency department.

\section{Mechanisms of} tachycardia.

An SVT usually has a QRS duration of $<120 \mathrm{~ms}$, unless there is bundle branch block (BBB) or an intraventricular conduction abnormality. The
QRS is narrow because ventricular activation occurs via the normal His-Purkinje system. SVT includes a wide range of arrhythmias that originate above the bifurcation of the bundle of His, such as sinus tachycardia, atrial flutter (AFL), atrial fibrillation (AF), atrial tachycardia (AT), atrioventricular junctional re-entrant tachycardia (AVJRT) and junctional ectopic tachycardia (JET). AVJRT can be due to atrioventricular nodal re-entrant tachycardia (AVNRT), where the antegrade limb is the slow pathway of the atrioventricular (AV) node and the retrograde limb the fast pathway, or atrioventricular re-entrant tachycardia (AVRT), where the antegrade limb is the AV node and the retrograde limb is the accessory pathway ('orthodromic' AVRT).
A WCT has a QRS duration $\geq 120 \mathrm{~ms}$ and may be caused by one of the following four mechanisms:

- VT. VT must be the default diagnosis in any patient with a WCT and accounts for $80 \%$ of all cases of $W C T{ }^{[1]}$ The presence of structural heart disease and a previous myocardial infarction (MI) increases the likelihood that the WCT is VT to $>90 \%{ }^{[2]} \mathrm{VT}$ usually occurs on the basis of a scar re-entrant mechanism (commonly due to a prior MI, cardiac surgery or cardiomyopathy). Some idiopathic VTs may occur in a structurally normal heart and be due to triggered activity (e.g. right ventricular outflow tract VT).

- SVT with a right or left BBB or an intraventricular conduction abnormality. This

\begin{tabular}{|c|c|}
\hline Regular & Irregular \\
\hline Narrow QRS & Narrow QRS \\
\hline $\begin{array}{l}\text { Sinus tachycardia } \\
\text { Atrial flutter } \\
\text { AVJRT (AVNRT, AVRT) } \\
\text { Atrial tachycardia } \\
\text { Junctional ectopic tachycardia }\end{array}$ & $\begin{array}{l}\text { Atrial fibrillation } \\
\text { Atrial flutter/tachy with variable } \\
\text { AVB } \\
\text { Multifocal atrial tachycardia }\end{array}$ \\
\hline $\begin{array}{l}\text { Ventricular tachycardia } \\
\text { SVT with BBB } \\
\text { Paced rhythm } \\
\text { Antidromic AVRT } \\
\text { Pre-excited SVT }\end{array}$ & $\begin{array}{l}\text { Atrial fibrillation with BBB } \\
\text { Atrial flutter, variable AVB + BBB } \\
\text { Pre-excited atrial fibrillation } \\
\text { Polymorphic ventricular tachycardia }\end{array}$ \\
\hline Regular $\quad$ Wide QRS & $\begin{array}{l}\text { Wide QRS Irregular } \\
\quad \text { Ire }\end{array}$ \\
\hline
\end{tabular}

Fig. 1. Classification of tachycardia (AVJRT = atrioventricular junctional re-entrant tachycardia; $A V N R T=$ atrioventricular nodal re-entrant tachycardia; $A V R T=$ atrioventricular re-entrant tachycardia; $B B B=$ bundle branch block; $A V B=$ atrioventricular block). 
accounts for $15-20 \%$ of WCTs. The BBB may be pre-existing or only apparent during the SVT, maintained by concealed retrograde trans-septal conduction (functional). Functional BBB occurs when an impulse conducts across the septum and blocks the contralateral bundle branch retrogradely, which results in BBB with the next conducted impulse.

- Pre-excited tachycardia. This accounts for $1-6 \%$ of all causes of WCT. Any SVT that conducts antegradely over an accessory pathway (Wolff-Parkinson-White syndrome) will produce a WCT. In 'antidromic' AVRT, the antegrade limb is the accessory pathway and the retrograde limb the AV node.

- Pacemaker tachycardia. Usually with RV apical pacing the morphology of the paced QRS complexes will have an atypical left bundle branch block (LBBB) pattern in V1 - V3, with left axis deviation. This may be seen in dual-chamber pacemakers (DDD) when there is tracking of the atrial rhythm (e.g. sinus tachycardia, atrial tachycardia). Pacemaker tachycardia may also occur in single-chamber pacemakers (VVIR) when the lower rate is increased by the sensor in the pacemaker.

\section{Diagnostic evaluation}

The evaluation of a patient with a tachycardia should begin with a detailed medical history (including medication history) and physical examination. The clinical context often helps in the interpretation of the 12-lead ECG

\section{History}

In a patient in whom a tachycardia is suspected but not confirmed, one should focus on the history of palpitations. In the emergency department, palpitations are most commonly due to sinus tachycardia. The following characteristics are important when taking a history, which may raise the suspicion of an underlying arrhythmia:

- Rate and regularity. The clinician should ask the patient to tap out the rhythm with his/ her fingers. In this way, the clinician can determine the nature of the palpitations, e.g. rapid regular, rapid irregular, slow regular, slow irregular, ectopic beats, missed beats or strong beats. Rapid regular beats are suggestive of an SVT or a VT. Rapid irregular beats are suggestive of AF or AFL with variable $\mathrm{AV}$ block. Ectopic beats or missed beats suggest premature ventricular complexes (PVCs) or premature atrial complexes (PACs).

- Mode of onset. Palpitations with an abrupt onset are suggestive of an SVT or a VT. A gradual onset or 'warm-up' is suggestive of sinus tachycardia.
- Mode of termination. Palpitations that end abruptly are suggestive of an SVT or a VT. A gradual termination or 'warmdown' is suggestive of sinus tachycardia. A tachycardia that terminates abruptly with a vagal manoeuvre, such as carotid sinus massage, confirms an AV nodal-dependent arrhythmia, i.e. AVNRT or AVRT, using an accessory pathway (Fig. 2).

- Circumstance or precipitating factors. Palpitations that occur at rest usually suggest SVT or VT. Palpitations that occur during exercise may be sinus tachycardia, but SVT or VT may be precipitated by exercise. Sinus tachycardia is a physiological response to sympathetic activation due to a pathological process (such as heart failure, thyrotoxicosis or sepsis). When sinus tachycardia occurs out of proportion to the physiological state, it is termed 'inappropriate' sinus tachycardia. This, however, is rare. Apparent inappropriate sinus tachycardia on ECG is more likely to be AFL with 2:1 block, the second flutter wave being hidden by the QRS.

- Associated symptoms. Palpitations may be associated with presyncope, syncope, chest pain or abnormal pulsations in the neck. Syncope may occur with a rapid SVT or VT, usually as a result of a fall in cardiac output, or after termination of the tachycardia because of overdrive suppression of the SA node.

- Associated heart disease. Underlying structural heart disease is an important substrate for the development of arrhythmias, e.g. a history of ischaemic heart disease or prior MI strongly suggests VT. Heart disease raises the suspicion of AF, AFL or VT. Lung disease may lead to atrial dilatation and AF, AFL or multifocal atrial tachycardia (MAT). A family history of sudden cardiac death or syncope should be sought when a familial cause is suspected (torsade de pointes with
A
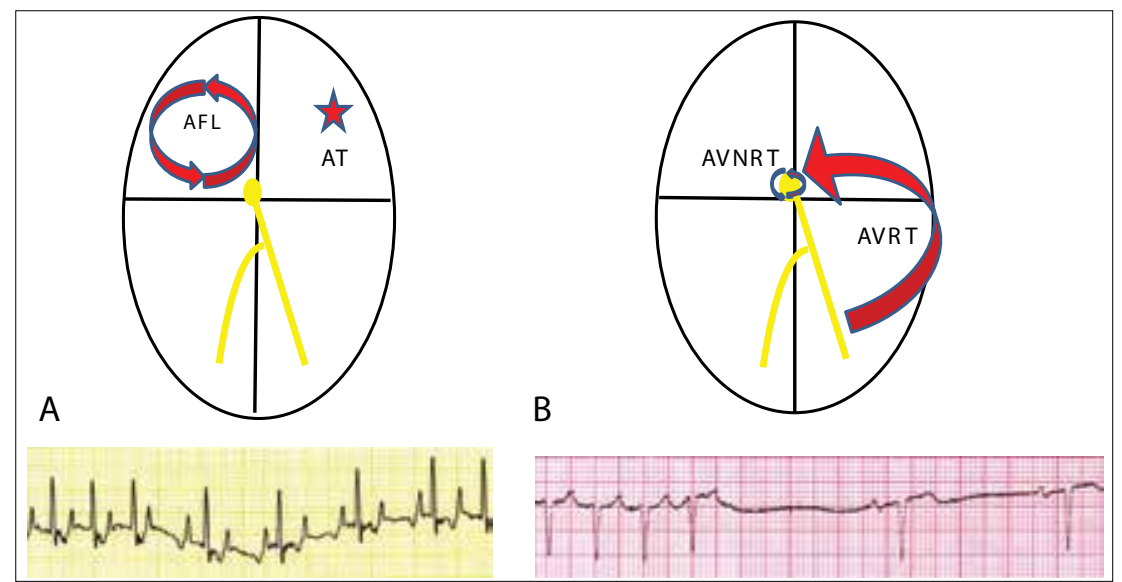

the congenital long QT syndrome and VT or PVCs with arrhythmogenic right ventricular cardiomyopathy (ARVC)). SVT usually occurs in a normal heart but can occur in patients with heart disease.

If a tachycardia is suspected, but has not been previously documented, the clinician has the option to arrange 24-hour Holter monitoring or inpatient telemetry, depending on the frequency of palpitations. In clinical practice, performing an ECG during an episode of future palpitations is likely to have the best yield. An implantable loop recorder can be considered if VT is strongly suspected.

\section{Physical examination}

The physical examination is useful to identify underlying cardiac disease that may serve as a substrate for arrhythmias. Signs of an underlying cardiomyopathy and heart failure raise the possibility that the palpitations are due to VT or AF/AFL. SVT can occur in patients with normal hearts and in those with underlying heart disease. Sometimes the SVT can cause left ventricular dysfunction, i.e. tachycardiainduced cardiomyopathy. The physical examination during a tachycardia is most helpful if signs of AV dissociation are present, which strongly suggests VT. Intermittent prominent cannon 'A' waves in the JVP waveform reflecting simultaneous atrial and ventricular contraction confirm AV dissociation.

The physician should look for clues to the cause of tachycardia by examining the 12-lead ECG in sinus rhythm. Prior ECGs should be sought and QRS morphology should be examined looking for BBB patterns and intraventricular conduction abnormalities

\section{Twelve-lead electrocardiography in sinus rhythm}

Fig. 2. Carotid sinus massage. (A) Atrial flutter or atrial tachycardia are AV nodal-independent tachycardias. Carotid massage causes transient $A V$ block, which reveals underlying $P$ waves ( $A F L=$ atrial flutter; $A T=$ atrial tachycardia). (B) AVJRT (AVNRT/AVRT) are AV nodal-dependent tachycardias. Carotid massage may terminate the tachycardia. 
(Fig. 3). The tachycardia and sinus rhythm QRS complexes may then be compared.

Some ECG abnormalities point towards structural heart disease, which may provide clues as to the most likely cause for an arrhythmia:

- Left atrial enlargement and/or PACs suggest the diagnosis may be AF or AFL.

- The presence of Q waves suggests prior MI and possible VT or AF/AFL.

- Marked left ventricular hypertrophy with deep septal Q waves in I, aVL and V4 - V6 suggests hypertrophic cardiomyopathy.

Other ECG abnormalities point towards a primary electrical cause for the arrhythmia:

- A short PR interval, delta wave and wide QRS complex are evidence of pre-excitation (WolffParkinson-White syndrome) and suggest a diagnosis of AVRT or pre-excited AF.

- Frequent PVCs with LBBB morphology and an inferior axis suggest right ventricular outflow tract VT as a cause, which may be idiopathic or due to ARVC. T-wave inversion in leads V1 - V3 and the presence of an Epsilon wave are further diagnostic signs of ARVC. ${ }^{[3]}$

- A long QT interval, Brugada pattern or early repolarisation pattern suggests polymorphic VT (PMVT) or torsade de pointes.

\section{Twelve-lead electrocardiography of the tachycardia}

The initial analysis of the tachycardia is best followed by a methodical approach to ECG interpretation. A high-quality 12-lead ECG is more useful in the clinical setting than individual rhythm strips.

The three main observations to consider in the ECG interpretation of a tachycardia are the following:

- Confirm the tachycardia (i.e. ventricular rate $\geq 100 \mathrm{bpm}$ ). The easiest way to calculate the ventricular rate is to count the number of QRS complexes and multiply by 6 (most ECGs are recorded at $25 \mathrm{~mm} / \mathrm{s}=$ 10-second recording).

- Are the QRS complexes wide ( $\geq 120 \mathrm{~ms})$ or narrow $(<120 \mathrm{~ms})$ ? Care must be taken to examine all leads, looking for the widest QRS complex. Some WCTs may have narrow QRS complexes in some leads.

- Are the QRS complexes regular or irregular?

The clinician is now able to classify the tachycardia into four groups, based on these three simple observations (Fig. 1):

- Regular, narrow complex tachycardias include sinus tachycardia, AT, AFL, AVJRT (AVNRT/AVRT) and JET.

- Irregular, narrow complex tachycardias include AF (most likely), AFL with variable block or MAT.
- Regular, wide complex tachycardias include monomorphic VT (in $80 \%$ of cases), SVT with right $\mathrm{BBB}(\mathrm{RBBB})$ or $\mathrm{LBBB}$, preexcited tachycardia over an accessory pathway, and pacemaker tachycardias.

- Irregular, wide complex tachycardias include PMVT, AF or AFL with RBBB or LBBB and pre-excited AF.

In patients with SVT, the next step would be to look for underlying $\mathrm{P}$ waves, flutter or fibrillary waves. There may be a $\mathrm{P}$ wave hidden in the T wave or QRS complex:

- P-wave morphology. P waves are usually best seen in the inferior leads (II, III and
aVF) and V1. The P-wave axis should be calculated (normal: $30-70^{\circ}$ ). $\mathrm{P}$ waves should be positive in the inferior leads because right atrial activation starts at the sinus node and propagates to the AV node in an inferior direction. By contrast, AVJRT (AVNRT or AVRT) or AT activates the atria in a superior direction, with negative $\mathrm{P}$ waves in the inferior leads (Fig. 4). These $\mathrm{P}$ waves tend to be narrow $(80 \mathrm{~ms})$ because both atria are depolarised simultaneously from the lower septum.

- Relative position of the $\mathrm{P}$ wave in the R-R interval. A common nomenclature used is to classify SVT as long RP (latter half)

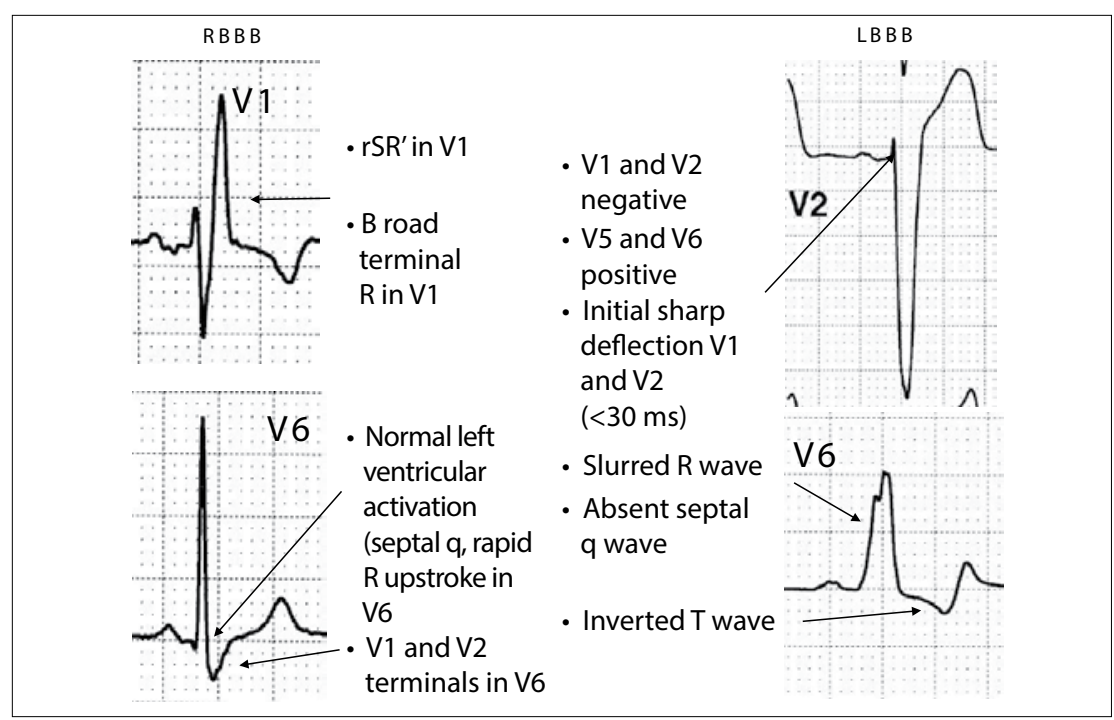

Fig. 3. Typical patterns of right and left $B B B$.

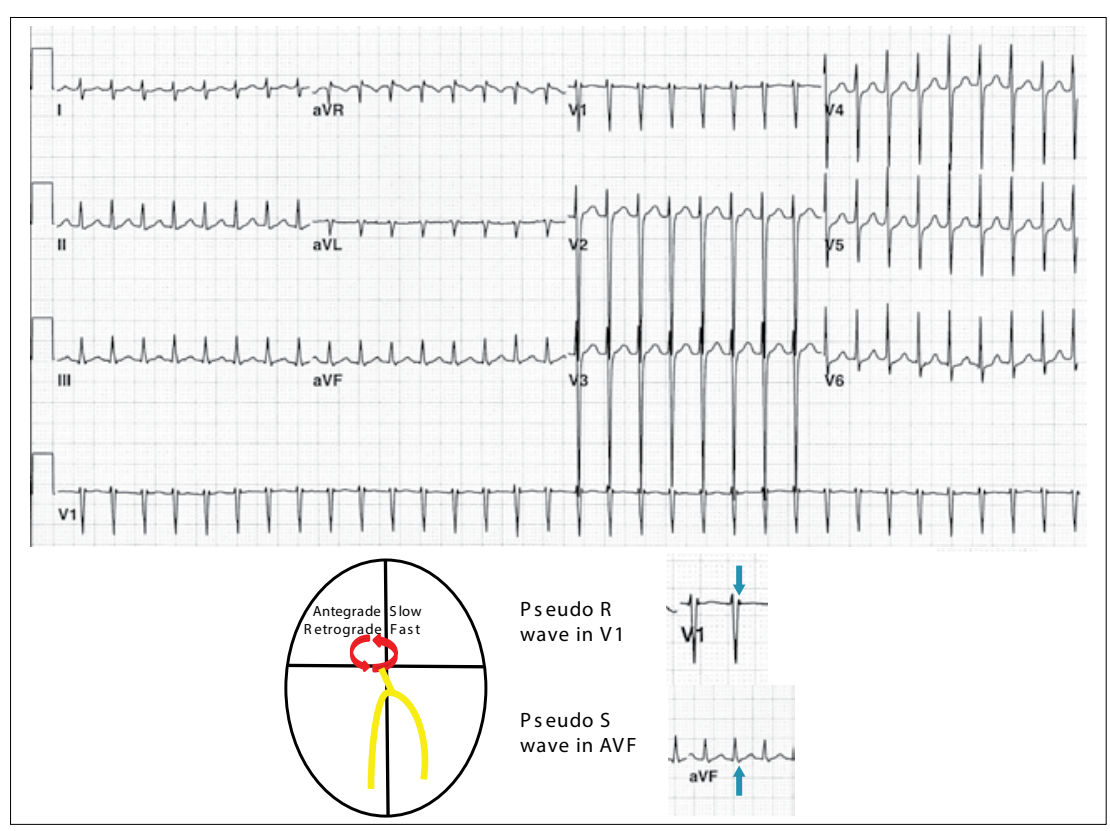

Fig. 4. An example of a narrow complex (supraventricular) tachycardia. $P$ waves are visible at the end of the QRS complexes. In V1, the P wave is seen as a pseudo R wave in V1 (arrow). In II, III and AVF the $P$ waves are negative and are seen as pseudo $S$ waves in AVF (arrow). In AVNRT the antegrade limb is the slow pathway and the retrograde limb the fast pathway of the AV node. 
or short RP tachycardias (first half), based on the position of the $\mathrm{P}$ wave in the $\mathrm{R}-\mathrm{R}$ interval. This is purely descriptive and does not help the clinician to narrow the differential diagnosis. Examples of a short RP tachycardia include: typical AVNRT, AVRT or AT. Examples of a long RP tachycardia include: atypical AVNRT, AVRT over a slowly conducting accessory pathway (also called permanent junctional reciprocating tachycardia) and AT. JET and AT can present as both types of examples. AVNRT (90\%) and AVRT (87\%) are usually short RP tachycardias, but ATs are usually long (11\%). ${ }^{[4]}$

- Onset and termination. If the SVT starts with a very long PR interval at the onset of the tachycardia, the clinician should diagnose AVNRT with antegrade slow pathway conduction accounting for the prolonged PR interval. An SVT that starts with a PVC is usually an AVJRT, and AT is improbable. SVT that terminates with a $\mathrm{P}$ wave is unlikely to be an AT, as the last atrial beat will probably not cause AV block with the same beat. It is more likely an AVJRT, where block in the AV node caused the tachycardia to terminate. A vagal manoeuvre increases vagal tone and may terminate an AV nodal-dependent tachycardia, but never an AT. For AFL and $\mathrm{AT}$, transient AV block may reveal underlying $\mathrm{P}$ waves or flutter waves (Fig. 2). A vagal manoeuvre commonly does not terminate an SVT and is thus unhelpful.

- Change in cycle length and morphology. The clinician should focus on any change in cycle length of the tachycardia. If a change in QRS rate changes the subsequent $\mathrm{P}$ wave (V drives A), AT is unlikely. QRS alternans (phasic alteration in the amplitude of the QRS complex) is a nonspecific finding in faster tachycardias, irrespective of the mechanism.

In patients with a WCT, looking for $\mathrm{P}$ waves is less important and the following needs to be examined:

- QRS morphology. As the main differential diagnosis of VT is an SVT with LBBB or RBBB, the clinician should examine whether the WCT has features of typical LBBB or RBBB. It is imperative that the clinician knows what typical RBBB and LBBB looks like (Fig. 3). In both RBBB and LBBB the initial activation of the QRS complex is rapid. If the width of the R wave in V1 and V2 is $>30 \mathrm{~ms}$ and/or the initial $\mathrm{R}$ wave in $\mathrm{V} 1$ and $\mathrm{V} 2$ to the nadir of the $\mathrm{S}$ wave is $>70 \mathrm{~ms}$, the patient is more likely to have VT (Fig. 5). ${ }^{[5]}$ Numerous algorithms have been published, distinguishing VT from SVT (the Brugada algorithm being the most widely cited) ${ }^{[6]}$
The algorithm is not useful to distinguish VT from pre-excited tachycardias and some have found it to be less accurate than that in the original publication. ${ }^{[7]}$

- Features specific for VT. AV dissociation, fusion beats and capture beats are specific for VT but are not sensitive. Negative concordance (i.e. all the QRS complexes in V1 - V6 are negative), in the absence of pacing, is specific for VT.

- Features of pre-excitation during sinus rhythm with QRS complexes similar to the WCT suggest a pre-excited tachycardia.

- Pacemaker spikes are not always visible, but the morphology will usually show atypical LBBB with left axis deviation and RV apical pacing.

\section{Management of tachycardia}

\section{Acute management of SVT}

The golden rule of $\mathrm{ABC}$ (airway, breathing, circulation) should be followed in the emergency management of SVT. If the patient is haemodynamically compromised, urgent direct current (DC) cardioversion should be performed. Most patients who present with an SVT are haemodynamically stable, allowing the physician to obtain a thorough history and do a physical examination.

The initial strategy should be to terminate an SVT with a vagal manoeuvre, such as carotid sinus massage, after excluding any carotid artery bruit. This should be performed with the patient in the supine position with a continuous 12-lead ECG. A vagal manoeuvre increases vagal tone and may terminate an
AVJRT (AVNRT/AVRT), but never an AT. For AFL and AT, transient AV block may reveal underlying $\mathrm{P}$ waves or flutter waves. If these manoeuvres do not terminate the SVT, the next step is intravenous adenosine. Starting doses of $6 \mathrm{mg}$ with doses of $12 \mathrm{mg}$ and $18 \mathrm{mg}$ are normally used. Adenosine has a short halflife ( $<10$ seconds) and produces transient heart block. Adenosine should be avoided in patients with an obvious AFL, as the reflex sympathetic activation may precipitate 1:1 AFL with haemodynamic decompensation. Adenosine will usually terminate AVJRT as well as some types of AT (making it less specific than carotid sinus massage). ${ }^{[8]}$ Intravenous verapamil should be avoided in the emergency setting.

The long-term management of SVT is medical or catheter ablation. Patients with recurrent symptomatic AVJRT can be treated with AV nodal blockers (such as beta-blockers or verapamil). Catheter ablation should be offered to patients with AVJRT as the success rate is high (95\%), with $5 \%$ requiring a second procedure. ${ }^{[9]}$ The decision to pursue a rate versus rhythm control strategy for AF/AFL should be individualised depending on symptoms, rate of tachycardia and whether there is underlying LV dysfunction.

\section{Acute management of WCT}

The golden rule of $\mathrm{ABC}$ should be followed in the emergency management of a WCT. If the patient is haemodynamically compromised, urgent DC cardioversion should be performed. It is not uncommon for a WCT to present in an unstable way,

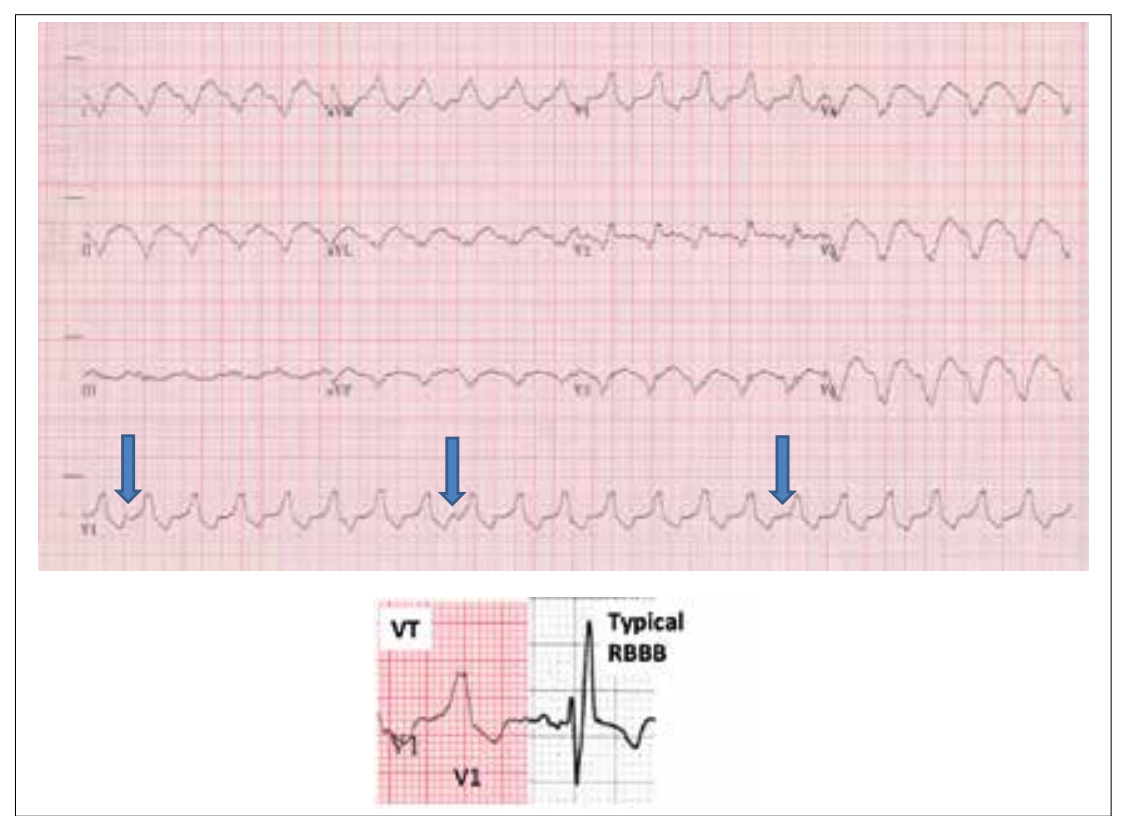

Fig. 5. An example of a ventricular tachycardia (WCT with a ventricular rate of $126 \mathrm{bpm}$ (regular)). Note: the morphology in V1 is not compatible with typical RBBB. There is AV dissociation (arrows show dissociated $P$ waves). 
irrespective of the cause. VT may degenerate into pulseless electrical activity and ventricular fibrillation (VF). As described above, a wide complex tachycardia should always be considered VT until proven otherwise. Haemodynamic stability does not exclude VT.

The treatment of choice for stable and unstable monomorphic VT is cardioversion after sedation has been administered. Amiodarone IVI is recommended in some guidelines as an initial strategy. ${ }^{[10]}$ Amiodarone was only $25 \%$ effective in terminating VT, with a sideeffect profile of hypotension that may worsen the haemodynamic status. ${ }^{[1]}$ Therefore, cardioversion should be readily available if amiodarone is chosen as an initial treatment strategy. Beta-blockers and verapamil are contraindicated in the acute setting and can cause severe haemodynamic deterioration and even VF or cardiac arrest. Patients with VT or WCT of uncertain origin should be referred to a cardiologist for further investigation and consideration of an implantable cardioverter defibrillator (ICD).

The treatment of PMVT or torsade de pointes due to a long QT interval (either congenital or more often acquired) is different from that of monomorphic VT. Electrical cardioversion is the treatment of choice. As the underlying cause of torsade de pointes is a long QT interval, most anti-

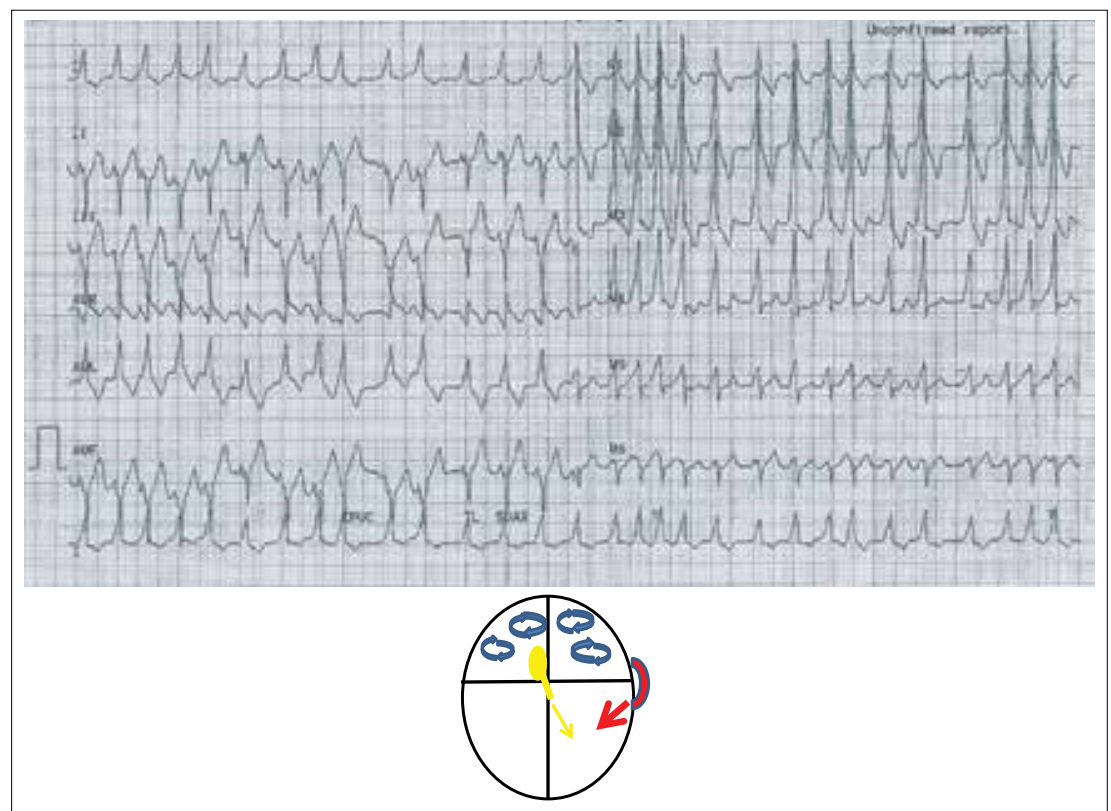

Fig. 6. An example of a wide complex irregular tachycardia. This is pre-excited AFL over a left lateral accessory pathway. Note that the QRS complexes are wide and vary in morphology because of variable degrees of fusion over the accessory pathway and the AV node. arrhythmic drugs (including amiodarone) will exacerbate this and are contraindicated. Once the patient has been stabilised, the underlying cause should be sought and treated (correct the hypokalaemia and hypomagnesaemia and discontinue all long QT-prolonging drugs). Acute ischaemia is a common cause of PMVT and urgent coronary angiography is indicated. Other causes of PMVT include channelopathies such as Brugada syndrome and catecholaminergic PMVT.

Some idiopathic outflow tract VTs may be adenosine sensitive. Adenosine should generally be avoided unless an SVT with $\mathrm{BBB}$ is suspected, as the reflex sympathetic activation may worsen VT. Verapamil must be avoided in all WCTs, with the rare exception of a patient with a known history of left fascicular VT proven to be verapamil sensitive in the past. Patients with idiopathic VT should be referred for ablation.

A WCT may suggest the diagnosis of pre-excited AF (Fig. 6). There is no role for medical therapy in the treatment of pre-excited AF, as beta-blockers, calcium channel blockers, adenosine and digoxin do not block and may enhance conduction over an accessory pathway, resulting in very rapid ventricular response with cardiac arrest. These patients should receive immediate electrical cardioversion and be

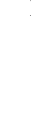

\section{References}

1. Issa ZF, Miller JM, Zipes DP. Clinical Arrhythmology and Electrophysiology. 2nd ed. Philadelphia, USA: Elsevier, 2012.

2. Tchou P, Young P, Mahmud R, Denker S, Jazayeri M Akhtar M. Useful clinical criteria for the diagnosis of ventricular tachycardia. Am J Med 1988;84(1):53-56. [http:// dx.doi.org/10.1016/0002-9343(88)90008-3]

3. Marcus FI, McKenna WJ, Sherrill D, et al. Diagnosis of arrhythmogenic right ventricular cardiomyopathy/ dysplasia: Proposed modification of the task force criteria. Circulation 2010;121(13):1533-1541. [http://dx.doi.org/10.1161/ CIRCULATIONAHA.108.840827]

4. Kalbfleisch SJ, el-Atassi R, Calkins H, Langberg JJ, Morady F. Differentiation of paroxysmal narrow QRS complex tachycardias using the 12-lead electrocardiogram. J Am Coll Cardiol 1993;21 (1):85-89. [http://dx.doi.org/10.1016/0735Cardiol 1993;2

5. Kindwall KE, Brown J, Josephson ME. Electrocardiographic criteria for ventricular tachycardia in wide complex left criteria for ventricular tachycardia in wide complex left
bundle branch block morphology tachycardias. Am bundle branch block morphology tachycardias. Am
Cardiol 1988;61(15):1279-1283. [http://dx.doi.org/10.1016/0002Cardiol 1988;61(15):12

9149(88)91169-1]
6. Brugada P, Brugada J, Mont L, Smeets J, Andries EW. A new

6. Brugada P, Brugada J, Mont L, Smeets J, Andries EW. A new
approach to the differential diagnosis of a regular tachycardia with a wide QRS complex. Circulation 1991;83(5):1649-1659. [http:// dx.doi.org/10.1161/01.CIR.83.5.1649]

7. Vereckei A, Duray G, Szénási G, Altemose GT, Miller JM. Application of a new algorithm in the differential diagnosis of wide QRS complex tachycardia. Eur Heart I 2007:28(5):589-600. [http://dx.doi.org/10.1093/eurhearti/ehl473]

8. Markowitz SM, Nemirovksy D, Stein KM, et al. Adenosineinsensitive focal atrial tachycardia: Evidence for de novo micro-reinsensitive focal atrial tachycardia: Evidence for de novo micro-re-
entry in the human atrium. J Am Coll Cardiol 2007;49(12):13241333. [http://dx.doi.org/10.1016/j.jacc.2006.11.037]

9. Fox DJ, Tischenko A, Krahn AD, et al. Supraventricular tachycardia: 9. Fox DJ, Tischenko A, Krahn AD, et al. Supraventricular tachycardia:
Diagnosis and management. Mayo Clin Proc 2008;83(12):1400Diagnosis and management. Mayo Clin Poc

10. Pedersen CT, Kay GN, Kalman J, et al. EHRA/HRS/APHRS expert consensus on ventricular arrhythmias. Europace 2014;16(9):12571283. [http://dx.doi.org/10.1093/europace/euu194]

11. Marill KA, deSouza IS, Nishijima DK, et al. Amiodarone or procainamide for the termination of sustaine stable ventricular tachycardia: An historical multicenter comparison. Acad Emerg Med 2010;17(3):297-230. [http://dx.doi. org/10.1111/j.1553-2712.2010.00680.x] 